\title{
Mass-sheet degeneracy: Fundamental limit on the cluster mass reconstruction from statistical (weak) lensing
}

\author{
M. Bradač ${ }^{1,2}$, M. Lombardi ${ }^{1,3}$, and P. Schneider ${ }^{1}$ \\ ${ }^{1}$ Institut für Astrophysik und Extraterrestrische Forschung, Auf dem Hügel 71, 53121 Bonn, Germany \\ e-mail: marusa@astro.uni-bonn.de \\ 2 Max-Planck-Institut für Radioastronomie, Auf dem Hügel 69, 53121 Bonn, Germany \\ 3 European Southern Observatory, Karl-Schwarzschild-Str. 2, 85748 Garching bei München, Germany
}

Received 25 November 2003 / Accepted 11 May 2004

\begin{abstract}
Weak gravitational lensing is considered to be one of the most powerful tools to study the mass and the mass distribution of galaxy clusters. However, weak lensing mass reconstructions are plagued by the so-called mass-sheet degeneracy the surface mass density $\kappa$ of the cluster can be determined only up to a degeneracy transformation $\kappa \rightarrow \kappa^{\prime}=\lambda \kappa+(1-\lambda)$, where $\lambda$ is an arbitrary constant. This transformation fundamentally limits the accuracy of cluster mass determinations if no further assumptions are made. We describe here a method to break the mass-sheet degeneracy in weak lensing mass maps using the distortion and redshift information of background galaxies and illustrate this by two simple toy models. Compared to other techniques proposed in the past, it does not rely on any assumptions on cluster potential; it can be easily applied to non-parametric mass-reconstructions and no assumptions on boundary conditions have to be made. In addition it does not make use of weakly constrained information (such as the source number counts, used in the magnification effect). Our simulations show that we are effectively able to break the mass-sheet degeneracy for supercritical lenses, but that for undercritical lenses the mass-sheet degeneracy is very difficult to break, even under idealised conditions.
\end{abstract}

Key words. dark matter - galaxies: clusters: general - gravitational lensing

\section{Introduction}

Galaxy clusters have been the focus of a very intense ongoing research in the past years. They are the most massive bound structures in the Universe; moreover, their large dynamical time scale allows them to retain information about their formation history. The mass and the mass distribution of galaxy clusters is particularly important for cosmological studies and therefore provides a critical test of the Cold Dark Matter (CDM) paradigm.

Weak gravitational lensing techniques are a unique tool to measure the projected mass of a cluster. This effect has long been recognized and measured (see Tyson et al. 1990), however it was only after the pioneering work of Kaiser \& Squires (1993; see e.g., Clowe \& Schneider 2001, 2002 for further applications) that the field began to flourish. Since then several other mass reconstruction techniques and applications of weak lensing have been developed (see e.g., Kaiser et al. 1995; Lombardi \& Bertin 1999; Seitz \& Schneider 1997; Bridle et al. 1998; Marshall et al. 2002; Hoekstra et al. 1998).

Unfortunately, these methods suffer from the fact that the projected surface mass density $\kappa$ can be determined only up to a degeneracy transformation $\kappa \rightarrow \kappa^{\prime}=\lambda \kappa+(1-\lambda)$, where $\lambda$ is an arbitrary constant. This invariance (first recognized by
Falco et al. 1985 in the context of strong lensing and by Schneider \& Seitz 1995 in the context of weak lensing) leaves the relation between the intrinsic and observed ellipticity unchanged. Therefore it cannot be broken by using only measurements of the distortion of the background sources, if these all lie at the same distance from the observer and fundamentally limits the accuracy of cluster mass determinations if no further assumptions are made.

A naive solution to this problem is to constrain $\lambda$ by making simple assumptions about $\kappa$. For example, one can assume that the surface mass density is decreasing with distance from the centre, implying $\lambda>0$. In addition, $\kappa$ is likely to be nonnegative, and so one can obtain an upper limit on $\lambda$ (for $\kappa<1$ ).

More quantitatively, with the use of wide field cameras one might try to assume that $\kappa \simeq 0$ at the boundary of the field, far away from the cluster center. However, if we consider for example a $M_{\text {vir }}=10^{15} M_{\odot}$ cluster at redshift $z=0.2$, we expect from $N$-body simulations to have a projected dimensionless density of about $\kappa \simeq 0.005$ at 15 arcmin from the cluster center (D. Clowe, private communication). Hence, even with the use of a $30 \times 30$ arcmin camera we expect to underestimate the virial mass of such a cluster by $\sim 20 \%$.

Another approach to determine the mass of a cluster from weak lensing data is to fit the shear signal with a parametric 
model (such as SIS or NFW). However, as shown in Clowe \& Schneider $(2001,2002)$, it is extremely difficult to distinguish SIS from NFW at high significance, and thus the mass estimates become difficult. They conclude that one of the main sources of uncertainty in determining the cluster mass and profile determination is the mass-sheet degeneracy.

Unfortunately, the mass-sheet degeneracy cannot be lifted by using the shapes of the background sources alone (Seitz \& Schneider 1997), and so one needs to make use of additional information. One promising solution to this problem is provided by the so-called magnification effect (see Broadhurst et al. 1995). Indeed, the local number counts of background sources is directly related to the magnification of the lens, which to the first order is given by $1+2 \kappa$. As a result, a careful estimate of the local density of background sources can lead to a direct measurement of the projected density $\kappa$ (see Fort et al. 1997; Taylor et al. 1998 for reported detections of this effect). In addition, other methods to measure cluster masses via magnification effects have been proposed (e.g. Bartelmann \& Narayan 1995 suggested to use the sizes of galaxies at a given surface brightness as a measure of the local lens magnification). All these methods, however, require a fine calibration of external parameters, such as the number counts of unlensed sources, which is very difficult to obtain with desired accuracy (Schneider et al. 2000).

In this work we will therefore focus on the possible methods to break the mass-sheet degeneracy by using distortion and redshift information of background sources. Seitz \& Schneider (1997) already considered this problem, and they showed that the mass-sheet degeneracy can be weakly broken provided that the probability distribution of the redshifts for the observed sources is known with good accuracy. Recently, it has been shown that photometric redshifts based on accurate multi-band photometry can be extremely accurate (see e.g. Benítez 2000). Hence, it is reasonable to assume that the individual redshifts of the background sources can be known in weak lensing studies. As we will show later, this in principle allows one to break the mass-sheet degeneracy.

This paper is organised as follows. In Sect. 2 we give the basic lens equations, introduce the notation we use, and introduce the problem of mass-sheet degeneracy. In Sect. 3 we focus on the cluster mass reconstruction technique and we describe the problem of mass-sheet degeneracy in more detail in Sect. 4. In Sect. 5 we describe the simulated data we are using and present the results. We conclude in Sect. 6.

\section{Statistical lensing preliminaries}

\subsection{Notation and basic lens relationships}

In this paper we use the standard weak lensing notation as described in Bartelmann \& Schneider (2001). We consider a lens with projected surface mass density $\Sigma(\boldsymbol{\theta})$, where $\boldsymbol{\theta}$ denotes the position in the lens plane. For a source at redshift $z$ and a lens at redshift $z_{\mathrm{d}}$ we define the critical surface mass density $\Sigma_{\mathrm{cr}}(z)$ as

$\Sigma_{\mathrm{cr}}=\frac{c^{2}}{4 \pi G} \frac{D_{\mathrm{s}}}{D_{\mathrm{d}} D_{\mathrm{ds}}}$, where $D$ represents the angular diameter distances; $D_{\mathrm{s}}=$ $D(0, z), D_{\mathrm{d}}=D\left(0, z_{\mathrm{d}}\right)$, and $D_{\mathrm{ds}}=D\left(z_{\mathrm{d}}, z\right)$ are the angular diameter distances between the observer and the source, the observer and the lens, and the lens and the source, respectively.

Since we will work with sources at different redshifts, we factorize the redshift dependence of the lens convergence $\kappa$ and of the shear $\gamma$ as

$\kappa(\boldsymbol{\theta}, z)=Z(z) \kappa(\boldsymbol{\theta}), \quad \gamma(\boldsymbol{\theta}, z)=Z(z) \gamma(\boldsymbol{\theta})$,

where $Z(z)$ is the so-called "cosmological weight" function:

$Z(z) \equiv \frac{\lim _{z \rightarrow \infty} \Sigma_{\mathrm{cr}}\left(z_{\mathrm{d}}, z\right)}{\Sigma_{\mathrm{cr}}\left(z_{\mathrm{d}}, z\right)} \mathrm{H}\left(z-z_{\mathrm{d}}\right)$.

The Heaviside step function $\mathrm{H}\left(z-z_{\mathrm{d}}\right)$ accounts for the fact that the sources that are closer to the observer than the deflector are not lensed. Note that, as suggested by its name, $Z(z)$ is cosmology dependent. For an Einstein-de Sitter cosmology it becomes

$Z(z)=\frac{\sqrt{1+z}-\sqrt{1+z_{\mathrm{d}}}}{\sqrt{1+z}-1} \mathrm{H}\left(z-z_{\mathrm{d}}\right)$.

In Lombardi \& Bertin (1999) cosmological weights were calculated for different cosmologies. The authors have shown that the differences between Einstein-de Sitter and the nowadays assumed standard cosmology (i.e. $\Omega_{\mathrm{m}}=0.3, \Omega_{\Lambda}=0.7$ ) are not significant for the purpose of cluster-mass reconstructions. Therefore we will from now on use Einstein-de Sitter cosmology. Similarly to $\kappa$ and $\gamma$, we define the redshift-dependent reduced shear $g(\boldsymbol{\theta}, z)$ as

$g(\boldsymbol{\theta}, z)=\frac{Z(z) \gamma(\boldsymbol{\theta})}{1-Z(z) \kappa(\boldsymbol{\theta})}$

Throughout this paper we use the complex ellipticity $\epsilon$ defined in terms of the second brightness moments $Q_{i j}$ as

$\epsilon \equiv \frac{Q_{11}-Q_{22}+2 \mathrm{i} Q_{12}}{Q_{11}+Q_{22}+2\left(Q_{11} Q_{22}-Q_{12}^{2}\right)^{1 / 2}}$

The transformation between the source ellipticity $\epsilon^{\mathrm{s}}$ and image ellipticity $\epsilon$ is given as a function of reduced shear $g(\boldsymbol{\theta}, z)$ (see Seitz \& Schneider 1997),

$\epsilon^{\mathrm{s}}= \begin{cases}\frac{\epsilon-g(\boldsymbol{\theta}, z)}{1-g^{*}(\boldsymbol{\theta}, z) \epsilon} & \text { for }|g(\boldsymbol{\theta}, z)| \leq 1, \\ \frac{1-g(\boldsymbol{\theta}, z) \epsilon^{*}}{\epsilon^{*}-g^{*}(\boldsymbol{\theta}, z)} & \text { for }|g(\boldsymbol{\theta}, z)|>1 .\end{cases}$

The inverse transformation is simply given by swapping $\epsilon^{\mathrm{s}}$ with $\epsilon$ and by substituting $-g$ for $g$. 
Under the assumption that the intrinsic ellipticity distribution is isotropic, $\left\langle\epsilon^{s}\right\rangle=0$, the expectation value for the image ellipticity at redshift $z$ becomes

$\langle\epsilon(z)\rangle= \begin{cases}g(\boldsymbol{\theta}, z) & \text { if }|g(\boldsymbol{\theta}, z)|<1, \\ \frac{1}{g^{*}(\boldsymbol{\theta}, z)} & \text { otherwise. }\end{cases}$

In the weak lensing approximation which we define by $\kappa \ll 1$, $|\gamma| \ll 1$ (thus $|g| \ll 1$ ) the expectation value is given by $\langle\epsilon(z)\rangle=$ $\gamma(\boldsymbol{\theta}, z)$.

\subsection{The problem of the mass sheet degeneracy}

In the simple case of background sources all having the same redshift, the mass-sheet degeneracy can be understood just using the above equations. Indeed, consider for a moment the transformation of the potential $\psi$

$\psi(\boldsymbol{\theta}, z) \rightarrow \psi^{\prime}(\boldsymbol{\theta}, z)=\frac{1-\lambda}{2} \boldsymbol{\theta}^{2}+\lambda \psi(\boldsymbol{\theta}, z)$,

where $\lambda$ is an arbitrary constant. $\kappa$ and $\gamma$ are related to the potential $\psi$ through its second partial derivatives (denoted by subscript), namely

$\kappa=\frac{1}{2}\left(\psi_{, 11}+\psi_{, 22}\right), \gamma_{1}=\frac{1}{2}\left(\psi_{, 11}-\psi_{, 22}\right), \gamma_{2}=\psi_{, 12}$.

From Eq. (9) it follows that $\kappa$ transforms as

$\kappa(\boldsymbol{\theta}, z) \rightarrow \kappa^{\prime}(\boldsymbol{\theta}, z)=\lambda \kappa(\boldsymbol{\theta}, z)+(1-\lambda)$,

and similarly the shear changes as $\gamma(\boldsymbol{\theta}, z)$ to $\lambda \gamma(\boldsymbol{\theta}, z)$. Therefore the reduced shear $g(\boldsymbol{\theta}, z)$ remains invariant.

Seitz \& Schneider (1997) have shown that in the case of a known redshift distribution, a similar form of the mass-sheet degeneracy holds to a very good approximation for non-critical clusters, i.e. for clusters with $|g(\boldsymbol{\theta}, z)| \leq 1$ for all source redshifts $z$. In such a case the standard weak-lensing mass reconstruction is affected by the degeneracy

$\kappa \rightarrow \kappa^{\prime} \simeq \lambda \kappa+\frac{(1-\lambda)\langle Z(z)\rangle}{\left\langle Z^{2}(z)\right\rangle}$,

where $\left\langle Z^{n}(z)\right\rangle$ denotes the $n$th order moment of the distribution of cosmological weights. As a result, standard weak-lensing reconstructions are still affected by the mass-sheet degeneracy even for sources at different redshifts; moreover, simulations show that the degeneracy is hardly broken even for lenses close to critical.

In this paper, we use the information of individual redshifts of background sources to break this degeneracy. As an illustration of the effect, suppose that half of the background sources are located at a known redshift $z^{(1)}$, and the other half at another known redshift $z^{(2)}$. Then, the weak lensing reconstructions based on the two populations will provide two different mass maps, $\kappa^{\prime}\left(\boldsymbol{\theta}, z^{(1)}\right)$ and $\kappa^{\prime}\left(\boldsymbol{\theta}, z^{(2)}\right)$, leading to two different forms of the mass-sheet degeneracy. In other words, the two mass reconstructions $(i=1,2)$ are given by

$\kappa^{\prime}\left(\boldsymbol{\theta}, z^{(i)}\right)=\lambda^{(i)} \kappa_{\mathrm{t}}\left(\boldsymbol{\theta}, z^{(i)}\right)+\left(1-\lambda^{(i)}\right)$ where we have denoted $\kappa_{\mathrm{t}}\left(\boldsymbol{\theta}, z^{(i)}\right)$ the true projected $\kappa$ of the lens at the angular position $\boldsymbol{\theta}$ for sources at redshift $z^{(i)}$. Since the transformation (13) holds for any $\boldsymbol{\theta}$, we have a system of equations to be solved for $\lambda^{(1)}$ and $\lambda^{(2)}$. The relation between $\kappa_{\mathrm{t}}\left(\boldsymbol{\theta}, z^{(1)}\right)$ and $\kappa_{\mathrm{t}}\left(\boldsymbol{\theta}, z^{(2)}\right)$ is known, namely from Eq. (2) follows

$\kappa_{\mathrm{t}}\left(\boldsymbol{\theta}, z^{(1)}\right) Z\left(z^{(2)}\right)=\kappa_{\mathrm{t}}\left(\boldsymbol{\theta}, z^{(2)}\right) Z\left(z^{(1)}\right)$.

Suppose one measures both $\kappa^{\prime}\left(\boldsymbol{\theta}, z^{(i)}\right)$ at $N$ different positions $\boldsymbol{\theta}_{j}$, this gives us a system of $2 N$ equations to be solved for $\lambda^{(i)}$ and $\kappa_{\mathrm{t}}\left(\boldsymbol{\theta}_{j}\right)$. The mass-sheet degeneracy is therefore at least in theory lifted.

It is interesting to observe that this argument only applies to relatively "strong" lenses. Indeed, for "weak" lenses, i.e. lenses for which we can use a first order approximation in $\kappa$ and $\gamma$, the expectation value of measured image ellipticities is $\langle\epsilon(z)\rangle=$ $\gamma(\boldsymbol{\theta}, z)$. In such case the degeneracy of the form

$\psi(\boldsymbol{\theta}, z) \rightarrow \psi^{\prime}(\boldsymbol{\theta}, z)=\frac{1-\lambda}{2} \boldsymbol{\theta}^{2}+\psi(\boldsymbol{\theta}, z)$

leaves the observable $\gamma(\boldsymbol{\theta}, z)$ unchanged. As a result, the method described above cannot be used to break the mass-sheet degeneracy for these lenses. Only when the $1-Z(z) \kappa$ term in the reduced shear becomes important and $g(\boldsymbol{\theta}, z)$ can be distinguished from $\gamma(\boldsymbol{\theta}, z)$ in the (noisy) data, will we be able to make unbiased cluster mass reconstructions.

The aperture mass measures (Kaiser 1995; Schneider 1996) are very convenient for measuring the weak lensing signal. A simple form of such measures is the so-called $\zeta$ statistics,

$\zeta\left(\boldsymbol{\theta} ; \vartheta_{1}, \vartheta_{2}\right) \equiv \bar{\kappa}\left(\boldsymbol{\theta} ; \vartheta_{1}\right)-\bar{\kappa}\left(\boldsymbol{\theta} ; \vartheta_{1}, \vartheta_{2}\right)$.

which gives the difference between the mean surface mass densities in a circle of radius $\vartheta_{1}$ at position $\boldsymbol{\theta}$ and in an annulus between the radii $\vartheta_{1}$ and $\vartheta_{2}$. It measures the mass contrast, and thus cannot be used to determine the absolute mass scale. Therefore despite the fact that it is not affected by the masssheet degeneracy transformation of the form Eq. (15), it will not be discussed further in this work.

In conclusion, using individual redshifts of background sources provides the most direct method to break the masssheet degeneracy. The simple argument sketched above for two redshift planes will therefore be considered in more detail in this paper and generalised to background galaxies at different redshifts. In the following we will use mock catalogues of image ellipticities, generated under optimistic assumptions. Such an approach will allow us to investigate the minimum lensing strength needed to be able to lift the degeneracy in forthcoming observations. As we will see below, this straightforward method can be successfully used to break the mass-sheet degeneracy in statistical lensing when applied in the strong lensing regime.

\section{Cluster mass reconstruction from image distortion}

\subsection{Maximum-likelihood approach}

To further investigate the effect of using individual redshifts in the cluster mass reconstructions, it is useful to adopt a Bayesian 
approach and to write the likelihood function of a given observed configuration of galaxy ellipticities.

Let us call $p_{\epsilon^{\mathrm{s}}}\left(\epsilon^{\mathrm{s}}\right)$ the probability distribution of intrinsic source ellipticities. For simplicity and better understanding we will here (and throughout this paper) assume the following truncated Gaussian distribution

$$
p_{\epsilon^{s}}\left(\epsilon^{s}\right)=\frac{1}{2 \pi \sigma^{2}\left[1-\exp \left(-1 / 2 \sigma^{2}\right)\right]} \exp \left(-\left|\epsilon^{s}\right|^{2} / 2 \sigma^{2}\right) .
$$

Note however that the results in this section are independent of the particular choice for $p_{\epsilon^{s}}$. The observed distribution $p_{\epsilon}(\epsilon \mid g)$ is related to the intrinsic one by (see Geiger \& Schneider 1998)

$p_{\epsilon}(\epsilon \mid g)=p_{\epsilon^{\mathrm{s}}}\left(\epsilon^{\mathrm{s}}(\epsilon \mid g)\right)\left|\frac{\mathrm{d}^{2} \epsilon^{\mathrm{s}}}{\mathrm{d}^{2} \epsilon}\right|(\epsilon \mid g)$.

The Jacobian determinant from the previous equation is given by

$\left|\frac{\mathrm{d}^{2} \epsilon^{\mathrm{s}}}{\mathrm{d}^{2} \epsilon}\right|(\epsilon \mid g)= \begin{cases}\frac{\left(|g|^{2}-1\right)^{2}}{\left|\epsilon g^{\star}-1\right|^{4}} & \text { for }|g| \leq 1, \\ \frac{\left(|g|^{2}-1\right)^{2}}{|\epsilon-g|^{4}} & \text { for }|g|>1 .\end{cases}$

In the weak lensing limit, $|g| \ll 1$, the Jacobian determinant is simply unity.

In general, the measured ellipticity $\epsilon^{\mathrm{m}}$ will differ from $\epsilon$ because of measurement errors:

$\epsilon^{\mathrm{m}}=\epsilon+\epsilon^{\mathrm{err}}$.

The error $\epsilon^{\mathrm{err}}$ is a random variable whose distribution depends on the details of the ellipticity measurement algorithm. In the following, for simplicity (and throughout this paper), we will assume that $\epsilon^{\mathrm{err}}$ is distributed according to a Gaussian distribution with dispersion $\sigma_{\text {err }}$. The actual shape measurement which corrects for PSF smearing (see e.g. Kaiser et al. 1995) can yield measured ellipticities with $\left|\epsilon^{\mathrm{m}}\right|>1$.

In our case, we write the probability distribution for the measured ellipticities as

$p_{\epsilon^{\mathrm{m}}}\left(\epsilon^{\mathrm{m}} \mid g\right)=\int p_{\epsilon}(\epsilon \mid g) p_{\epsilon^{\mathrm{err}}}\left(\epsilon-\epsilon^{\mathrm{m}}\right) \mathrm{d} \epsilon$,

where $p_{\epsilon^{\mathrm{er}}}\left(\epsilon^{\mathrm{err}}\right)$ is the probability distribution of measurement errors. This convolution takes into account the abovementioned measurement errors and therefore we do not need to discard galaxies with $\left|\epsilon^{\mathrm{m}}\right|>1$.

The likelihood function $\mathcal{L}$ is the product of probability densities for observed image ellipticities $\epsilon_{i}^{\mathrm{m}}$, and depends on the model parameters $\pi$ through the reduced shear at the image positions $\left\{g_{i}\right\}$ :

$\mathcal{L}(\pi)=\prod_{i=1}^{N_{\mathrm{g}}} p_{\epsilon^{\mathrm{m}}}\left(\epsilon_{i}^{\mathrm{m}} \mid g_{i}\right)$.

Here $N_{\mathrm{g}}$ is the number of observed galaxies with measured ellipticities $\epsilon_{i}^{\mathrm{m}}$. It is more convenient to deal with the $\log$ likelihood function, defined as

$l(\pi) \equiv-\ln \mathcal{L}(\pi)=-\sum_{i=1}^{N_{g}} \ln p_{\epsilon}\left(\epsilon_{i} \mid g\right)$.
By minimising $l(\pi)$ we obtain the most likely parameters $\pi_{\max }$ given the observations.

For each of the $N_{\mathrm{g}}$ galaxies we need to calculate the probability distribution $p_{\epsilon^{\mathrm{m}}}\left(\epsilon^{\mathrm{m}} \mid g\right)$ (cf. Eq. (21)) of measured image ellipticity $\epsilon_{i}^{\mathrm{m}}$ given the reduced shear $g$. The evaluation of the full likelihood function is therefore non-trivial, since $N_{\mathrm{g}}$ integrals need to be calculated. Thus, we are dealing with two Gaussian probability distributions for source ellipticities $\epsilon^{\mathrm{s}}$ and the measurement error $\epsilon^{\mathrm{err}}$. However, the final measured errors for observed image ellipticities $\epsilon^{\mathrm{m}}$ is not distributed according to a Gaussian in general, since the source ellipticities are first being lensed and only then the measurement errors are added (i.e. the Jacobian determinant in Eq. (18) is not unity).

If the reduced shear is small (i.e. for undercritical lenses), this problem can be solved by including the measurement errors in the source probability distribution $p_{\epsilon}$ : in other words, if $|g| \ll 1$, the Jacobian determinant is unity and $p_{\epsilon}(\epsilon \mid g)=$ $p_{\epsilon^{s}}\left(\epsilon^{\mathrm{s}}(\epsilon \mid g)\right)$. We can therefore interchange the convolution with $p_{\epsilon_{\text {err }}}$ appearing in Eq. (21) with the lensing transformation of Eq. (18). The calculations are then trivial, since $p_{\epsilon^{\mathrm{s}}}$ and $p_{\epsilon^{\mathrm{err}}}$ can be taken to be Gaussians with dispersions $\sigma_{\epsilon^{s}}^{2}$ and $\sigma_{\text {err }}^{2}$ respectively; hence, we can just use for $p_{\epsilon^{s}}$ a Gaussian with dispersion $\sigma_{\epsilon^{s}}^{2}+\sigma_{\text {err }}^{2}$.

This is, however, not the case for critical lenses. As we show latter, the approximate form for the measured ellipticities (cf. Eq. (18)) gives biased results for lenses with a large fraction of background sources having $|g| \simeq 1$. For the same reason, we should not discard galaxies with $\left|\epsilon^{\mathrm{m}}\right|>1$ without properly accounting for their removal in Eq. (21). Therefore, unless otherwise stated, we will use the probability distribution for measured ellipticity given in Eq. (21). It still does not account for the measurement errors on the redshifts of background sources, however these are less important.

\section{2. $\chi^{2}$ approach}

Finally, we note an alternative to the maximum-likelihood approach, namely a chi-square fitting. In our case, the $\chi^{2}$ function can be written as

$\chi^{2}(\pi)=\sum_{i=1}^{N_{g}} \frac{\left|\epsilon_{i}-\langle\epsilon\rangle\right|^{2}}{\sigma_{i}^{2}}$

where

$\sigma_{i}^{2}=\left(1-|\langle\epsilon\rangle|^{2}\right)^{2} \sigma_{\epsilon^{s}}^{2}+\sigma_{\text {err }}^{2}$.

Unfortunately, simulations show that such an approach does not give an unbiased result for critical lenses. This is due to two main reasons. First, the expression (25) is only an approximation for the true variance, that contains higher (even) order moments of the distribution of $\epsilon^{\mathrm{s}}$ (Lombardi 2000). Second and more important, ellipticity errors are not normally distributed for lenses with large reduced shears, $|g| \simeq 1$ (see Geiger \& Schneider 1998, Fig. 5). In the maximum likelihood approach, on the other hand, the real distribution of errors is properly accounted for and in the asymptotic limit the method will therefore give an unbiased result. Hence, in the following we will only discuss the likelihood approach. 


\section{The mass-sheet degeneracy}

From the discussion above, it is apparent that only galaxies with $|g| \simeq 1$ contribute significantly to the removal of the mass-sheet degeneracy for two reasons:

- the dispersion on the observed ellipticities is proportional to $\left(1-\left|\langle\epsilon\rangle^{2}\right|\right)^{2}$ (cf. Eq. (25)), and so galaxies with $|g| \simeq 1$ provide more accurate estimates of the local reduced shear;

- recalling the argument discussed after Eq. (13), galaxies with $|g| \ll 1$ present a simple mass-sheet degeneracy (cf. Eq. (15)) which cannot be broken using the method described here. Therefore one needs the information from $|g| \simeq 1$ galaxies to break the degeneracy.

It is important to realize that the likelihood function in the way we wrote it already takes into account the first point mentioned above. As a result, galaxies with $|g| \simeq 1$ are effectively more important for the purpose of breaking the mass-sheet degeneracy, while those with $|g| \ll 1$ do not contribute at all (as sketched in Sect. 2.2). It is sensible, therefore, to take into account this point when writing the approximate form of the mass-sheet degeneracy presented in Eq. (12). In other words, we assume the following transformation of $\left\langle Z^{n}(z)\right\rangle$

$\left\langle Z^{n}(z)\right\rangle \rightarrow\left\langle Z^{n}(z)\right\rangle^{\prime}=\frac{\sum_{i=1}^{N_{\mathrm{g}}} w_{i} Z_{i}^{n}}{\sum_{i=1}^{N_{\mathrm{g}}} w_{i}}$,

where $w_{i}$ are the weight factors and for $w_{i}=$ const. we recover the results of Seitz \& Schneider (1997). For giving more weights to galaxies with $|g| \simeq 1$, we will however use $w_{i}=$ $1 / \sigma_{i}$, defined in Eq. (25). As we mentioned before this is only an approximation of the true dispersion, however as we will show latter it is adequate to describe the approximate masssheet degeneracy behaviour.

\section{Simulated data}

To test whether we can break the mass-sheet degeneracy by using redshift information on the background sources, we performed two simple tests on simulated data.

We considered first the most simple model possible, a constant sheet of mass with external shear (Family I). More precisely, we set $\kappa(\boldsymbol{\theta})=C_{1}, \gamma_{1}(\boldsymbol{\theta})=C_{2}$, and $\gamma_{2}(\boldsymbol{\theta})=C_{3}$, with $C_{i}$ constants. Thus, in this model the convergence and shear for each galaxy depend only on the redshift of the source and not on its position. Although, clearly, this model is completely unrealistic, it is very useful as a test of our method. Indeed, it is reasonable to assume that if we are not able to break the mass-sheet degeneracy in this simple and favorable situation, it is unlikely that we will be able to break it in more realistic and complicated cases. This simple model can also be used as an indicator of how strong the lens should be in order to obtain a reliable estimate for the average surface mass density $\kappa$ across the field.

The second family we used is a non-singular model that approximates an isothermal sphere for large distances (see Schneider et al. 1992) in which we allow for a constant sheet in surface mass density (hereafter Family II). The dimensionless surface mass density is given by

$\kappa\left(\theta / \theta_{\mathrm{c}}\right)=\kappa_{0} \frac{1+\theta^{2} /\left(2 \theta_{\mathrm{c}}^{2}\right)}{\left(1+\theta^{2} / \theta_{\mathrm{c}}^{2}\right)^{3 / 2}}+\kappa_{1}$,

where $\kappa_{0}, \kappa_{1}$ are dimensionless constants and $\theta_{\mathrm{c}}$ is the core radius.

We used the following recipe to generate mock catalogues of background sources:

1. 2000 galaxies were drawn randomly across the field of $6 \times$ $6 \operatorname{arcmin}^{2}$, thus giving a density of 55 galaxies $\operatorname{arcmin}^{-2}$.

2. The intrinsic ellipticities $\epsilon^{\mathrm{s}}$ were drawn according to Eq. (17) characterized by $\sigma=\sigma_{\epsilon^{s}}=0.15$.

3. We draw the redshifts of the background sources, following Brainerd et al. (1996) from a gamma distribution

$p_{\mathrm{z}}(z)=\frac{z^{2}}{2 z_{0}^{3}} \exp \left(-z / z_{0}\right)$,

with $z_{0}=2 / 3$; the mean redshift is $\langle z\rangle=3 z_{0}=2$, and the mode is $z_{\text {mode }}=2 z_{0}=4 / 3$. The corresponding cosmological weights were evaluated assuming an Einstein-de Sitter cosmology. We put the lens at a redshift $z_{\mathrm{d}}=0.2$. For the purpose of mass-sheet degeneracy breaking one might think it would be an advantage to have a higher redshift lens, e.g. $z_{\mathrm{d}}=0.4$. In such a case most of the galaxies would lie on the steep part of the function $Z(z)$ and therefore we would have a higher scatter of $Z_{i}$ values. However, this effect is compensated for by the fact that the average reduced shear is lower for a lens at higher redshift. The effect of the number change of background sources further favours the low redshift cluster, however not significantly.

4. For each galaxy, we evaluated the local shear according to the lens model, its position and redshift; then we lensed the galaxy ellipticities. Note that the positions of the source galaxies were not transformed, i.e. we neglected here the magnification effect of the lens.

5. The measurement error $\epsilon^{\mathrm{err}}$ on the observed ellipticities was drawn from the distribution (17) with $\sigma=\sigma_{\text {err }}=0.1$ and added to the lensed ellipticities.

6. In most cases we considered measurement errors on the redshifts of the galaxies to simulate the use of photometric redshifts. These errors were drawn from a Gaussian distribution with $\sigma_{\text {zerr }}=0.06\left(1+z_{i}\right)$ (see Benítez 2000); in adding the errors we ensured that the resulting redshifts are always positive.

In generating the mock catalogues we have tried to simulate an ideal case, since we are trying to answer the question of when (and if at all) the mass-sheet degeneracy can be broken in statistical lensing mass reconstructions. From a comparison between independent observations of the same galaxies in the COMBO-17 survey, $\sigma_{\text {err }} \simeq 0.1-0.15$ (for each component of the ellipticity) can be estimated (Kleinheinrich 2003). The typical values of $\sigma_{\epsilon^{s}}$ are optimistically $\simeq 0.2$. However even a less optimistic case $\left(\sigma_{\epsilon^{\mathrm{s}}}=0.3, \sigma_{\text {err }}=0.15\right)$ does not change our conclusions. 


\subsection{Results of the model fitting to the simulated data}

For each set of true model parameters $\pi_{\mathrm{t}}$ we generated 100 mock catalogues. Using these, we searched for the most likely parameters $\pi_{\max }$ by minimising the log-likelihood function (23). We performed the minimisation with C-minuit (James \& Roos 1975), a routine which is a part of the CERN Program Library. C-minuit is designed to minimise a multi-parameter function and analyse its shape around the minimum. It simultaneously makes use of the gradient as well as downhill simplex method.

Despite the goodness of the routine, one has to be very careful when performing the minimisation, as the function (23) has logarithmic singularities for $|g|=1$ when $\sigma_{\text {err }}=0$ is assumed. If, for a particular parameter set, a background galaxy happens to have $|g| \simeq 1$, the log-likelihood function (23) diverges and the minimisation procedure has difficulties in "climbing" over such a region, possibly leading to secondary minima.

In the case of $\sigma_{\text {err }} \neq 0$ this is less likely to happen, as the convolution with $p_{\epsilon^{\text {err }}}$ in Eq. (21) is effectively smoothing the probability distribution. Unfortunately the integration in Eq. (21) has to be performed numerically. The noise resulting from numerical calculations is therefore present in the loglikelihood function and the minimisation routine is not able to search for a minimum, since the function is not smooth. On top of that, the integrations are CPU consuming; one needs to perform them for each galaxy separately. To avoid these problems, we note that function $p_{\epsilon^{\mathrm{m}}}(21)$ depends on three parameters (without a loss of generality we can assume $g$ to be real and accordingly transform $\left.\epsilon^{\mathrm{m}}\right)$. We therefore evaluate $p_{\epsilon^{\mathrm{m}}}$ on a three-dimensional grid (with GNU Scientific Library qags routine, http: //www.gnu.org/software/gsl) and use trilinear interpolation to evaluate the log-likelihood.

Finally, we stress that the model fitted to the data belonged, for all simulations, to the same family as the original model used to generate the galaxy catalog. Although unrealistic, this assumption allows us to directly compare the deduced parameters with the original ones, and thus simplifies the evaluation and interpretation of the simulation results. The free parameters of both models are $\pi=\kappa, \gamma_{1}, \gamma_{2}$ for Family I, $\pi=\kappa_{0}, \kappa_{1}$ for Family II. These correspond to the mass sheet degeneracy transformation, for the case where redshift information is not known; $\kappa(\boldsymbol{\theta}) \rightarrow \kappa^{\prime}(\boldsymbol{\theta})=\lambda \kappa(\boldsymbol{\theta}, z)+(1-\lambda)$ and $\gamma(\boldsymbol{\theta}) \rightarrow \gamma^{\prime}(\boldsymbol{\theta})=$ $\lambda \gamma(\boldsymbol{\theta})$. By investigating the distribution of best fit parameters $\pi$ for both models, we can answer the question of how strong the lens needs to be to lift the abovementioned degeneracy under idealised conditions.

\subsubsection{Family I}

Mock catalogues for Family I were generated using $\gamma_{1}=\gamma_{2}=$ 0.1 (see Fig. 1, panel a) and $\gamma_{1}=\gamma_{2}=0.2$ (panel b); 10 different values of $\kappa$ ranging from 0.5 to 1.5 were chosen for both sets. For each model we fitted 100 mock catalogues (generated as described above) and we marked with crosses the resulting best fit parameters (the original values of the parameters are represented as squares). To explore the importance of redshift measurement errors we have generated catalogues without and with redshift errors as described above (in Fig. 1 we show only the latter).

Surprisingly, even for a relatively strong lens with $\kappa=0.5$ we are not able to break the mass-sheet degeneracy with good accuracy. For this model, the relative error on $\kappa$ is of the order of $20 \%-40 \%$, which should be considered very large, given the idealized conditions used in our simulations. Hence, the results of our simulations suggest that it is very difficult to break the mass-sheet degeneracy for non-critical lenses using shape information only. As expected, for data without redshift errors the fit slightly improves, although not substantially.

On the other hand, for critical and close to critical lenses, we are effectively able to break the mass-sheet degeneracy. The surface mass density is well constrained for $\kappa \gtrsim 1$. As expected, the results show that galaxies with $|g| \simeq 1$ contribute substantially to the removal of the degeneracy. If the redshift uncertainty is not added the fit improves and the constraints are tighter.

Finally, it is interesting to observe that in some cases (e.g. $\kappa=1$ and $|\gamma|=0.14$ ) we obtain biased results if we use $p_{\epsilon}$ instead of $p_{\epsilon^{\mathrm{err}}}$ for calculating log-likelihood (see Fig. 2). Namely, in Fig. 2a we use the likelihood function that implies first convolving the data with $p_{\epsilon^{\text {err }}}$ and only then performing the lensing transformation (see discussion after Eq. (23)). This can severely bias results for lenses where many galaxies have $|g| \simeq 1$. Note that here the expected mass-sheet degeneracy calculated using $w_{i}=1 / \sigma_{i}$ in Eq. (26) needs to be calculated with $\sigma_{\text {err }}=0$, as we are not correctly accounting for the measurement errors in this case.

In addition to the global mass sheet invariance, there is a local point invariance. Namely, looking at a single image ellipticity we cannot distinguish between $g$ and $1 / g^{*}$ (Schneider \& Seitz 1995). This invariance is in practice easily broken, either by assuming a profile or simply by stating that $\kappa$ increases towards the cluster centre. In the case of all sources being at the same redshift, for the Family I, however, since $\kappa=$ const. this is a global invariance. In such a case the log-likelihood function has two equivalent minima, one corresponding to $g$ and one to $1 / g^{*}$. However since we do have the redshift information, this invariance is broken in most cases.

For our special case with $\kappa=1$ and $|\gamma|=0.14$ this is not so simple. The average absolute value of reduced shear for the galaxies is $\langle|g|\rangle \simeq 1$ and therefore for most galaxies the two minima lie close together. If the probability distribution of lensed ellipticities is properly accounted for, the resulting solutions give an unbiased estimate for the true minimum (see Fig. 2b). If this is not the case, as in Fig. 2a, the results can be biased.

This special case was presented as an extreme example. In practice such a lens is of course unrealistic. However we want to stress that critical lenses need to be treated with caution when using statistical lensing.

\subsubsection{Family II}

Mock catalogues for Family II were generated using $\kappa_{0}=0.9$, $\theta_{\mathrm{c}}=1.5$ arcmin, and 4 different values of $\kappa_{1}$ ranging from 

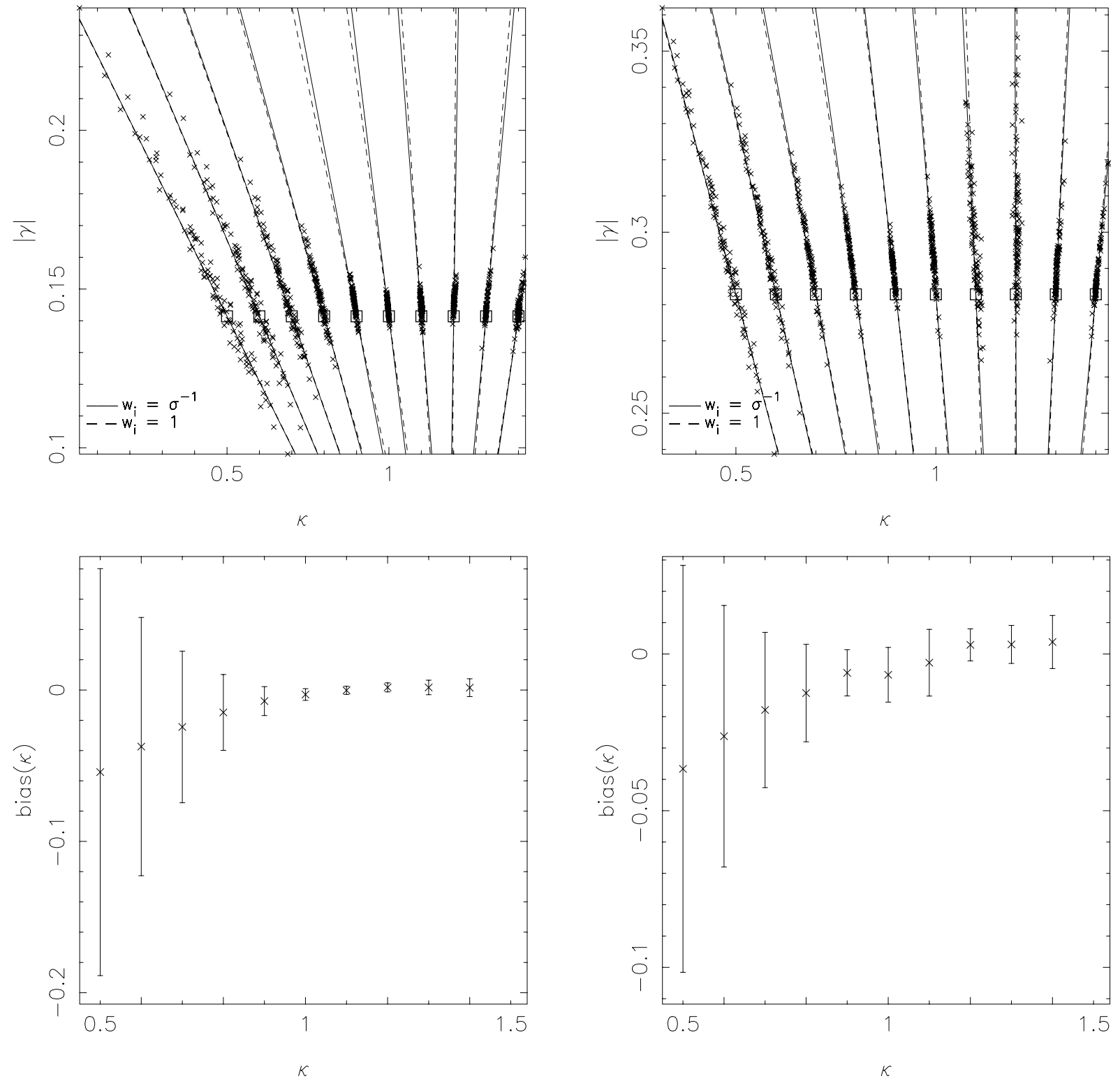

(a)

(b)

Fig. 1. Top: recovered parameter values (crosses) as a result of minimising the log-likelihood function (23). For each of the ten sets of parameters (panel a) $\gamma_{1}=\gamma_{2}=0.1$, panel b) $\gamma_{1}=\gamma_{2}=0.2$ ) - denoted by squares -100 mock catalogues were created using model Family I and the same family was used to fit the data. We use 3 free parameters $\left(\kappa, \gamma_{1}\right.$, and $\left.\gamma_{2}\right)$ for fitting, here we plot $\kappa$ and $|\gamma|$. Lines correspond to the expected mass-sheet degeneracy calculated using the weighting scheme given by $w_{i}=1 / \sigma_{i}$ (solid lines) and $w_{i}=$ const. (dashed lines) in Eq. (26). Bottom: bias and variance for the recovered values of $\kappa$ for the data described above.

0.2 to 0.5 . We fitted these data with the same family of models and using $\kappa_{0}$ and $\kappa_{1}$ as free parameters; note that we fixed the core radius to the same value as the original profile. Figure $3 \mathrm{a}$ shows the results of log-likelihood minimisation for the data with added redshift errors. Solid and dashed lines in the figure correspond to the expected mass-sheet degeneracy calculated using the weighting of $w_{i}=1 / \sigma_{i}$ and $w_{i}=$ const. in Eq. (26) respectively; dot-dashed lines give $\kappa_{0}+\kappa_{1}=$ const.

Finally we plot the bias and the variance of the recovered values for $\kappa_{1}$ in Fig. $3 b$. When the lens becomes critical the errors are small enough and we are able to constrain $\kappa_{1}$ with high accuracy. For these lenses we are therefore effectively able to break the mass-sheet degeneracy. This result is in accordance with the conclusions from the constant-lens model.

Surprisingly, for the case $\kappa_{0}=0.9$ and $\kappa_{1}=0.2$ we do not see the expected mass-sheet degeneracy, rather, the best-fit parameters lie along the line $\kappa_{0}+\kappa_{1}=$ const. Likely, this is due to the fact that for this model we have galaxies with $|g| \simeq 1$ close to the centre, where the surface mass density is $\kappa(0)=\kappa_{0}+\kappa_{1}$. Since the galaxies with $|g| \simeq 1$ are the ones that contribute most in breaking the mass-sheet degeneracy, it is not surprising for this model to see a degeneracy along the line $\kappa_{0}+\kappa_{1}=$ const. 


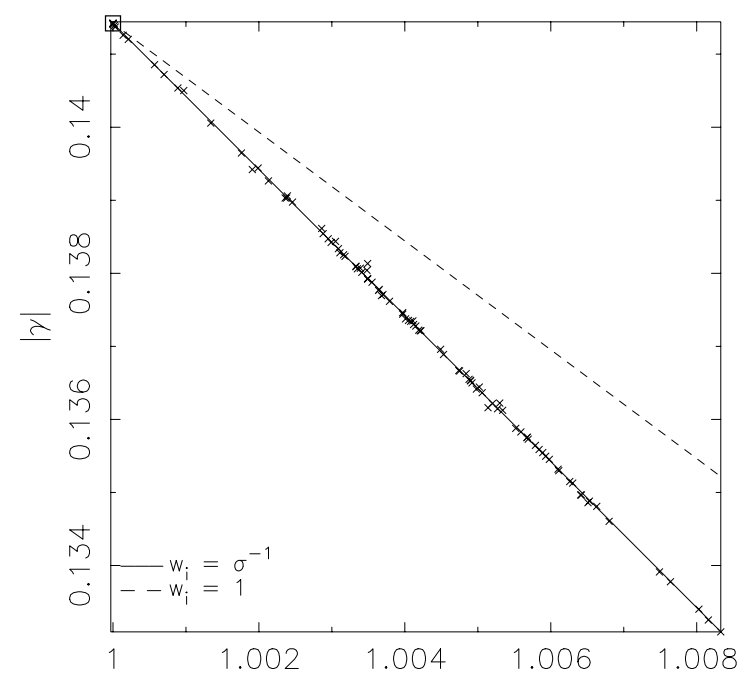

(a)

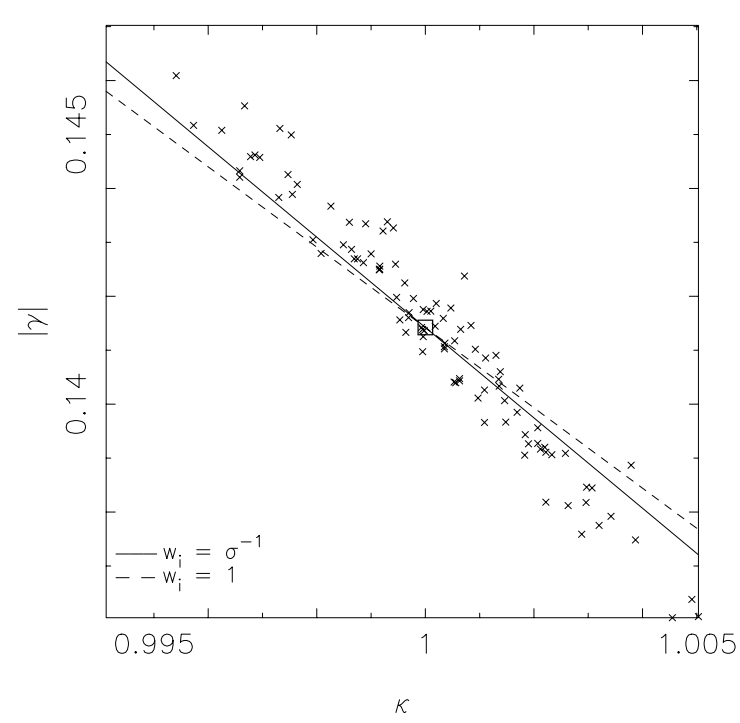

(b)

Fig. 2. Recovered parameter values (crosses) as a result of minimising the log-likelihood function Eq. (23). Panel a) shows results obtained using $p_{\epsilon}(\epsilon \mid g)$ - Eq. (18) - for the observed probability distribution for ellipticities, while for the panel b) we use (as in the rest of this paper) $p_{\epsilon^{\mathrm{m}}}\left(\epsilon^{\mathrm{m}} \mid g\right)$ - Eq. (21). For the parameter set $\kappa=1.0, \gamma_{1}=\gamma_{2}=0.1-$ denoted by a square -100 mock catalogues were created using model Family I and the same family was used to fit the data. Redshift errors were not added. We use 3 free parameters $\left(\kappa, \gamma_{1}\right.$, and $\left.\gamma_{2}\right)$ for fitting, here we plot $\kappa$ and $|\gamma|$. Lines correspond to the expected mass-sheet degeneracy calculated using Eq. (26). We used the weighting scheme given by $w_{i}=$ const. for the dashed lines. For the solid lines $w_{i}=1 / \sigma_{i}$ was used, however $\sigma_{\text {err }}=0$ was employed for panel a) only. This is due to the fact that we are using $p_{\epsilon}(\epsilon \mid g)$ in a) for the observed probability distribution for ellipticities, rather than $p_{\epsilon^{\mathrm{m}}}\left(\epsilon^{\mathrm{m}} \mid g\right)$.

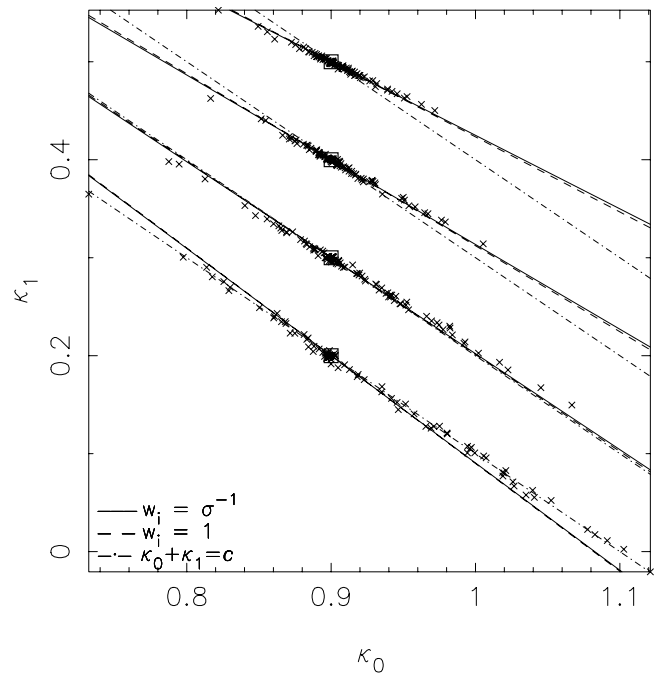

(a)

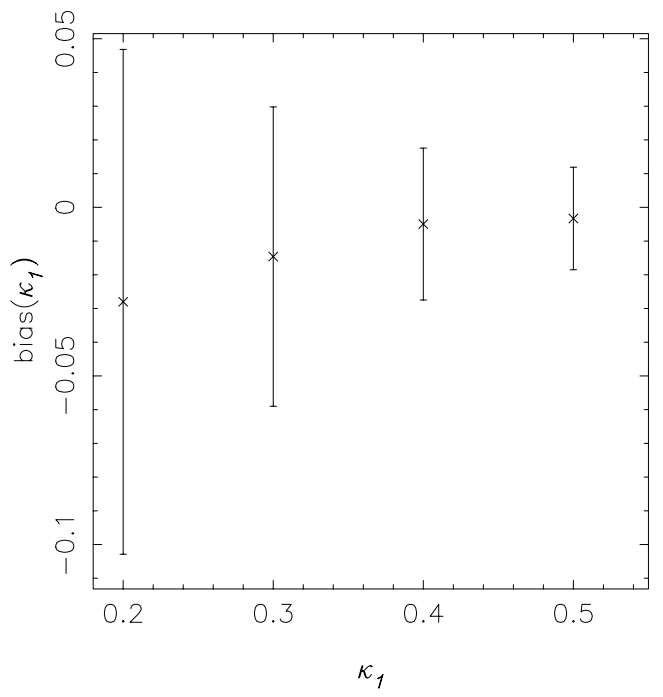

(b)

Fig. 3. a) Recovered parameter values (crosses) as a result of minimising the log-likelihood function Eq. (23). For each of the four sets of parameters (denoted by squares) 100 mock catalogues were created. We use model Family II and the same family was used to fit the data. The model has 2 free parameters $\left(\kappa_{0}\right.$ and $\left.\kappa_{1}\right)$ - see Eq. (27). Solid and dashed lines correspond to the expected mass-sheet degeneracy calculated using $w_{i}=1 / \sigma_{i}$ and $w_{i}=$ const. in Eq. (26) for the weighting scheme respectively (both almost overlap). Dot-dashed lines are given by $\kappa_{0}+\kappa_{1}=$ const. b) Relative bias and variance for the recovered values of $\kappa_{1}$.

If we take $\theta_{\mathrm{c}}$ to be a free parameter in the fitting, we obtain the degeneracy $\kappa_{0}+\kappa_{1}=$ const for all four sets of model parameters $\pi_{\mathrm{t}}$. Since $\theta_{\mathrm{c}}$ is allowed to vary, it can adjust so that the resulting best-fit model has, for lower values of $\kappa_{0}$, lower values of $\theta_{\mathrm{c}}$. The region of $|g| \simeq 1$ is then approximately unchanged as long as $\kappa_{0}+\kappa_{1}=\kappa_{0 \mathrm{t}}+\kappa_{1 \mathrm{t}}$.

\subsection{Ensemble-averaged log-likelihood}

Using the log-likelihood function (23) we can in principle obtain the best fitting parameters $\pi_{\max }$ given the observations and the confidence regions on these parameters. To obtain the expected errors of parameters obtained from a single 
realization of data, one can calculate the ensemble-averaged log-likelihood. Ensemble-averaging also provides a useful test for the behaviour of log-likelihood function in the asymptotic limit. Given the redshift distribution of the background sources $p(z)$ and following Schneider et al. (2000) we write the ensemble-average in the general form as

$\langle l\rangle(\pi)=n_{\mathrm{g}} \int \mathrm{d}^{2} \theta \int \mathrm{d}^{2} \epsilon p_{\epsilon}(\epsilon \mid g) \int \mathrm{d} z p_{z}(z) l(\pi)$,

where $n_{\mathrm{g}}$ gives the number of galaxies per unit area, and $p_{\epsilon}(\epsilon \mid g)$ is the probability distribution of lensed ellipticities calculated using lens parameters $\pi_{\mathrm{t}}$.

Asymptotically (i.e. when the number of source galaxies $N_{\mathrm{g}}$ is very large), the quantity $2 \Delta l$, where

$\Delta l=\langle l\rangle(\pi)-\langle l\rangle\left(\pi_{\mathrm{t}}\right)$

behaves as a random variable following a $\chi_{M}^{2}$ distribution, where $M$ is the number of free parameters. In particular, for Family I, where $M=3$, we expect $68.3 \%, 90 \%, 95.4 \%$, and $99 \%$ points within the levels $2 \Delta l=\{3.53,6.25,8.02,11.2\}$, while for Family II $(M=2)$ the corresponding levels are $2 \Delta l=\{2.30,4.61,6.17,9.21\}$. It is interesting to test the behavior of this quantity and the accuracy of the asymptotic limit in our case.

Note that, as shown by Geiger \& Schneider (1998), the ellipticity distribution is generally skewed even for relatively small $(|g|=0.6)$ reduced shears; hence, we need to evaluate all integrations of Eq. (29), including the one on $\epsilon$, numerically. The integration has been carried out using the GNU Scientific library routines.

In Fig. 4 we show an example of the ensemble-average log-likelihood for one set of simulated parameters for model Family II; the other sets give similar results. We simulated the data using parameters $\kappa_{0}=0.9$ and $\kappa_{1}=0.2$ without adding the redshift errors. This is the extreme case mentioned above, where the distribution of recovered parameters lies in a very narrow valley along the line where $\kappa_{0}+\kappa_{1}=$ const. (dash-dotted line) rather than along the expected mass-sheet degeneracy line (solid line and dashed line for different weighting schemes). The ensemble-average log-likelihood calculations confirm the anomalous behavior of the degeneracy.

The $2 \Delta l=9.21$ contour in Fig. 4 should enclose $99 \%$ of all points. This is not satisfied in our case; this is mainly due to the fact that we are not in the regime of the asymptotic limit (seen through the fact that the points in the plot are not distributed according to a Gaussian distribution).

\section{Conclusions}

In this paper we considered a new method to break the masssheet degeneracy in weak lensing mass reconstructions using shape measurements only. A detailed analysis of this method has also clearly shown that breaking the mass-sheet degeneracy is very difficult even in optimal conditions; arguably, it is extremely difficult in normal observational conditions.

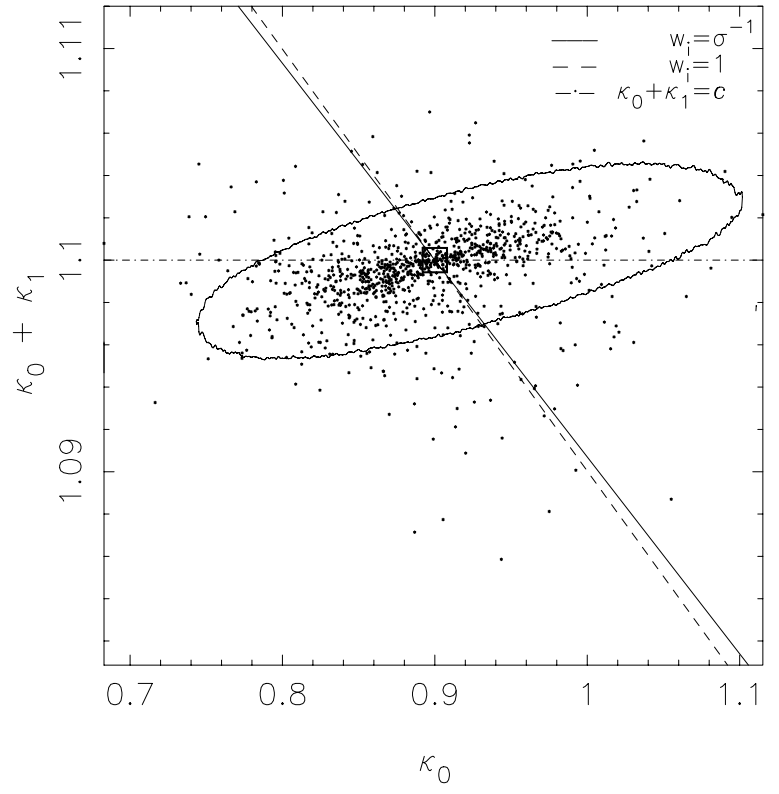

Fig. 4. Ensemble-averaged log-likelihood for model Family II with $\kappa_{0}=0.9$ and $\kappa_{1}=0.2$ (square). Contour gives the difference of ensemble-averaged log-likelihoods with a level of $2 \Delta l=9.21$. In contrast to previous figures 1000 recovered model parameters are plotted as dots and $\kappa_{0}+\kappa_{1}$ vs. $\kappa_{1}$ is plotted (note the small range on $y$ axis). The solid line gives the expected mass-sheet degeneracy line calculated with $w_{i}=1 / \sigma_{i}$ in Eq. (26) and the dashed line is calculated with $w_{i}=$ const. (both almost overlapping). The dash-dotted line is given by $\kappa_{0}+\kappa_{1}=$ const.

\section{Our main conclusions are:}

1. The mass-sheet degeneracy can be broken by using the redshift information of the individual sources. However, this is effective for critical clusters only, i.e. for clusters that have sizable regions where multiple imaging is possible (and thus perhaps observed). The statistical lensing analysis has to be extended close to and inside the critical curves of the cluster. In the regions far outside the critical curve, where weak lensing mass reconstructions are normally performed, the lens is too weak for the mass-sheet degeneracy to be broken by using redshift and distortion information only, even when idealised conditions are employed.

2. Using simulations we find that the correlation remaining from the mass-sheet degeneracy transformation for critical lenses is well described by Eq. (12), $\kappa \rightarrow \kappa^{\prime} \simeq \lambda \kappa+(1-$ $\lambda)\langle Z(z)\rangle /\left\langle Z^{2}(z)\right\rangle$, where the moments of the cosmological weights are calculated using $w_{i}=1 / \sigma_{i}$ in (26).

3 . In order to break the mass-sheet degeneracy with current data it is necessary to extend the statistical lensing analysis closer to the cluster centre and to simultaneously perform a weak and strong lensing analysis of the cluster. This will be a subject of future study.

We are developing a method which combines weak and strong lensing mass reconstruction techniques simultaneously in a non-parametric fashion. Weak and strong lensing data has been previously combined by e.g. Kneib (2003); however, only the combination of weak lensing signal on scales $>500 \mathrm{kpc}$ with the strong lensing signal at $\sim 100 \mathrm{kpc}$ was taken into 
account. Our approach to breaking the mass-sheet degeneracy can be employed also for non-parametric mass reconstruction. The non-parametric statistical lensing reconstruction technique needs to be extended to and within the critical regions, resulting in breaking the mass-sheet degeneracy in practice. Such a method does not rely on any assumption of the parametric form of the potential.

In summary, although breaking the mass-sheet degeneracy has proven to be surprisingly difficult, we have shown that it is in principle possible if one combines constraints on different scales (note that we assumed the knowledge of the cluster-mass profile; in practice one obtains the profile by using standard weak-lensing mass reconstructions). The mass-sheet degeneracy is probably the most severe limit of current weak lensing mass reconstructions, and is generally responsible for a significant fraction of the final error on the "total" mass of the cluster. Hence, breaking the mass-sheet degeneracy in practice is one of the most important challenges of weak lensing studies of clusters in the near future.

Acknowledgements. We would like to thank Douglas Clowe and Oliver Czoske for many useful discussions that helped improve the paper. This work was supported by the International Max Planck Research School for Radio and Infrared Astronomy at the University of Bonn, by the Bonn International Graduate School, and by the Deutsche Forschungsgemeinschaft under the project SCHN 342/3-1.

\section{References}

Bartelmann, M., \& Narayan, R. 1995, ApJ, 451, 60

Bartelmann, M., \& Schneider, P. 2001, Phys. Rep., 340, 291
Benítez, N. 2000, ApJ, 536, 571

Brainerd, T. G., Blandford, R. D., \& Smail, I. 1996, ApJ, 466, 623

Bridle, S. L., Hobson, M. P., Lasenby, A. N., \& Saunders, R. 1998, MNRAS, 299, 895

Broadhurst, T. J., Taylor, A. N., \& Peacock, J. A. 1995, ApJ, 438, 49

Clowe, D., \& Schneider, P. 2001, A\&A, 379, 384

Clowe, D., \& Schneider, P. 2002, A\&A, 395, 385

Falco, E. E., Gorenstein, M. V., \& Shapiro, I. I. 1985, ApJ, 289, L1

Fort, B., Mellier, Y., \& Dantel-Fort, M. 1997, A\&A, 321, 353

Geiger, B., \& Schneider, P. 1998, MNRAS, 295, 497

Hoekstra, H., Franx, M., Kuijken, K., \& Squires, G. 1998, ApJ, 504, 636

James, F., \& Roos, M. 1975, Computer Phys. Commun., 10, 343

Kaiser, N. 1995, ApJ, 439, L1

Kaiser, N., \& Squires, G. 1993, ApJ, 404, 441

Kaiser, N., Squires, G., \& Broadhurst, T. 1995, ApJ, 449, 460

Kleinheinrich, M. 2003, Ph.D. Thesis, University of Bonn

Kneib, J. 2003, ApJ, 598, 804

Lombardi, M. 2000, Ph.D. Thesis

Lombardi, M., \& Bertin, G. 1999, A\&A, 342, 337

Marshall, P. J., Hobson, M. P., Gull, S. F., \& Bridle, S. L. 2002, MNRAS, 335, 1037

Schneider, P. 1996, MNRAS, 283, 837

Schneider, P., Ehlers, J., \& Falco, E. 1992, Gravitational Lenses (Berlin, Heidelberg, New York: Gravitational Lenses, Springer-Verlag)

Schneider, P., King, L., \& Erben, T. 2000, A\&A, 353, 41

Schneider, P., \& Seitz, C. 1995, A\&A, 294, 411

Seitz, C., \& Schneider, P. 1997, A\&A, 318, 687

Taylor, A. N., Dye, S., Broadhurst, T. J., Benitez, N., \& van Kampen, E. 1998, ApJ, 501, 539

Tyson, J. A., Wenk, R. A., \& Valdes, F. 1990, ApJ, 349, L1 